J. KSIAM Vol.16, No.2, 137-149, 2012

\title{
An efficient algorithm to measure the insurance risk of casuality insurance company using VaR methodology
}

\author{
JOON HWA BAN ${ }^{1}$, HYUN CHEOL HWANG ${ }^{2}$, AND HOSAM KI ${ }^{3 *}$
}

${ }^{1}$ FNPRICING INC., SOUTH KorEA

E-mail address: jhban®406@yahoo.com, jhban@fnpricing.com

${ }^{2}$ GARgoyle Group, USA

E-mail address: hchwang77@gmail.com

${ }^{3}$ KIS PRICING INC., SOUTH KOREA

E-mail address: hosamki@gmail.com, kihs@kispricing.com

\begin{abstract}
We propose an efficient method to measure the insurance risk of causality insurance companies by using the CreditRisk+ methodology. This method is superior to previous methods in several aspects. Its computation speed is very fast and the input data form is simple. It is able to aggregate both credit risk and insurance risk, so the insurance company can manage the risk in combined manner. In this paper, we propose a mathematical method to obtain the aggregate loss distribution of portfolios having correlation among products or business lines as a general case, and then suggest its implementation algorithm. Finally we apply this method to the real data from Korea Insurance Development Institute (KIDI) and discuss its availability to real applications.
\end{abstract}

\section{INTRODUCTION}

The insurance company has several kinds of risks which result from the type of insurance business transacted. The International Association of Insurance Supervisors (IAIS) classifies risks of insurance company into Insurance risk, Asset risk and other risks. According to IAIS, "the insurance risks (equivalently, technical risks) represent the various kinds of risk that are directly or indirectly associated with the technical or actuarial bases of calculation for premiums and technical provisions in both life and non-life insurance, as well as risks associated with operating expenses and excessive or uncoordinated growth. ... They differ depending on the class of insurance. Insurance risks exist partly due to factors outside the company's area of business activities, and the company often may have little influence over these factors. The effect of such risks - if they materialise - is that the company may no longer be able to fully meet the guaranteed obligations using the funds established for this purpose, because either the claims frequency, the claims amounts, or the expenses for administration and settlement are higher than expected."

Received by the editors November 8 2011; Revised May 10 2012; Accepted in revised form May 302012.

2010 Mathematics Subject Classification. 91G80.

Key words and phrases. Insurance risk, VaR methodology, credit risk

*Corresponding author 
The insurance risk is a kind of intrinsic risk which is more difficult to measure than other risks. The development and implementation of efficient mathematical algorithms to measure exact insurance risks are necessary and urgent for introducing RBC (risk based capital) solvency margin system to Korean insurance industry. In order to measure insurance risk in some manner and apply it to solvency margin system for insurance company we need to estimate a loss distribution for each product or each business line firstly, to aggregate the loss distributions considering correlations among them secondly, so to have an aggregate loss distribution finally. We propose an efficient methodology to estimate an aggregate loss distribution for measuring the insurance risk in this paper.

The estimation of the aggregate loss distribution, the first and essential step for measuring the risks of insurance company, enables the insurance company to calculate VaRs(Value at Risk) according to each business line, each asset class, and each liability, and finally the firm-wide aggregated VaR which results in measuring the economic capital or the RBC solvency margin. And we can calculate the appropriate risks which will be prepared for future, by generating the future loss distribution using the aggregated current loss distribution combined with scenario analysis or stress test or DFA (Dynamic Financial Analysis).

The estimation of loss distribution has been studied by several researchers in traditional actuarial science. Heckman and Meyers (1983) estimated the severity and the frequency of each business lines, exactly the characteristic functions of them using the collective risk theory, and got the aggregated loss distribution by convolution. Robertson (1992) adapted the same manner and proposed a more efficient convolution method, discrete Fourier transform, DFT). The convolution methods have been done under the assumption of independent business lines, but there exists correlations among them in real cases. You can see how to estimate the correlated aggregate loss distribution in Wang (1998).

Such traditional methods, however, have several shortcomings. The estimation of loss distribution is made either by parametric methods or by nonparametric method. It is wellknown that the parametric method cannot capture the rare event probability, especially for loss distribution and the nonparametric demands too much times. Even the worse, those traditional methods cannot meet practical needs in measuring the aggregated risks of market risks, credit risks, and insurance risks and so on. The usual measuring methodologies of market, and credit risk have been developed in terms of VaR, and the insurance risk measured by those traditional ones cannot be aggregated into VaR framework.

In this paper, we suggest a methodology to overcome those shortcomings and to help the insurance company manage the insurance risk in view of aggregated risk management with market, and credit risks because it belongs into VaR framework. Its algorithm is modified from that in CreditRisk+. Furthermore, our algorithm is simple to implement and fast to estimate the loss distribution.

CreditRisk + is a credit risk measuring methodology to estimate the loss distribution of credit portfolio which consists of loans, default able bonds and so on. It is being used by many financial institutions all over the world for measuring credit VaRs and managing credit risks since its algorithm has been offered firstly by CSFB (Credit Suiss First Boston). And it adapts a nonparametric and analytic approximation to the whole probability distribution so as to be fast in estimating a loss distribution. This is why various kinds of financial 
institutions have adopted it as a method to measure credit risks. As results it has been well developed for practical applications of creditVaR in capital allocation and Risk-Adjusted Performance Measurement (RAPM). We study how to apply the CreditRisk+ methodology to measure the insurance risk of causality insurance company in this paper. And we sample a real loss data set classified according to business lines from Korea Insurance Development Institute to apply our method and to estimate the loss distribution coming from insurance risks.

Our method has good points when insurance companies manage the firm-wide risk in view of the aggregated risk management.

First, they can get the aggregated VaR when they use our method to measure their insurance risks and CreditRisk + to measure their credit risks. It is of consequence to the insurance company which has individual loan portfolios because only CreditRisk + is applicable for those portfolios.

Second, our algorithm is so fast even though it is nonparametric. As we mentioned above, our algorithm is a kind of nonparametric and analytic approximation which overcomes the shortcoming of nonparametric methods-the slow converging rate. Another nonparametric methods demand us too much computing times to get a desirable error bound.

Third, the input data format to our risk-measuring model is very simple. Our model assumes the multivariate normal distribution for severity and we estimate correlations between each business lines using serial data of severities to plug them into our model in simple manner.

Fourth, our model loosens the assumption of independence among business lines which is taken in CreditRisk+. The independent sectors assumption in CreditRisk + is too strict for practitioners to meet, so that they may hesitate to apply the estimating VaR to capital allocation and RAPM because they ignore the assumption of independent sectors to a certain extent.

This paper is composed as the followings; we will explain CreditRisk + methodology briefly in Chapter 2. In Chapter 3 we will explain in detail how to apply CreditRisk + to estimate the loss distribution combining severity and frequency in each case of independent business lines and correlated business lines, and in Chapter 4, two mathematical algorithms to estimate the loss distribution: the recursive method and the FFT method. In Chapter 5 we will apply our algorithm to real data set from Korea Insurance Development Institute to get the aggregated loss distribution. We will conclude in Chapter 6.

\section{CREDIT RISK + METHODOLOGY}

In this chapter we explain the CreditRisk+ methodology (CSFP, 1997) briefly. We consider a portfolio exposed to credit risks by $K$ obligors. We assume that all obligors are affected by $N$ risk factors $S=\left(S_{1}, S_{2}, \ldots, S_{N}\right)$. Let $p_{A}$ and $\tau_{A}$ be the expectation and the standard deviation of default probability for obligor $A$ and let $w_{A k}$ be the weight

of risk factor $S_{k}$ for obligor $A$. Such weights have to satisfy $0 \leq w_{A k} \leq 1, \sum_{k=1}^{N} w_{A k} \leq 1$. Thus 
$\sum_{k=1}^{N} w_{A k}$ specifies the systematic risk for obligor $A$, while $w_{A 0}=1-\sum_{k=1}^{N} w_{A k}$ represents the weight of idiosyncratic risk, which is independent of risk factors $S=\left(S_{1}, S_{2}, \ldots, S_{N}\right)$, of obligor $A$.

Consider the potential loss $\varepsilon_{A}$ for obligor $A$. It is one of the features of CreditRisk+ to work with discretized losses. For this purpose we fix a loss unit $L_{0}$ and choose a positive integer $v_{A}$ as a rounded version of $\varepsilon_{A} / L_{0}$. Then the aggregate portfolio loss in CreditRisk + as a multiple of the basic loss unit $L_{0}$ is given by

$$
X=\sum_{A} v_{A} D_{A}
$$

with $D_{A}$ being Poisson distributed random variables with stochastic intensities

$$
p_{A}^{S}=p_{A}\left(w_{A 0}+\sum_{k=1}^{N} w_{A k} S_{k}\right)
$$

conditional on independent gamma distributed random variables $S=\left(S_{1}, S_{2}, \ldots, S_{N}\right)$. Then we can obtain the expectation $\mu_{k}$ and the standard deviation $\sigma_{k}$ for $S_{k}, k=1,2, \cdots, N$

$$
\mu_{k}=\sum_{A} w_{A k} p_{A}, \quad \sigma_{k}=\sum_{A} w_{A k} \tau_{A} .
$$

We introduce the portfolio polynomial of the $k$-th factor to be

$$
Q_{k}(z)=\sum_{A} w_{A k} p_{A} z^{\nu_{A}}, \quad k \in\{0,1,2, \cdots, N\} .
$$

Then the PGF(probability generating function) of the CreditRisk + model can be expressed in closed analytic form

$$
G(z)=\exp \left[\left(Q_{0}(z)-Q_{0}(1)\right)-\sum_{k=1}^{N} \frac{\mu_{k}^{2}}{\sigma_{k}^{2}} \ln \left\{1-\frac{\sigma_{k}^{2}}{\mu_{k}^{2}}\left(Q_{k}(z)-Q_{k}(1)\right)\right\}\right] .
$$

On the other hand, from the definition of the PGF of a discrete, integer-valued random variable, we know that $G(z)$ may also be represented as

$$
G(z)=\sum_{j=0}^{\infty} P(X=j) z^{j} .
$$

Thus the central problem is the efficient and numerically stable computation of the probabilities $P(X=j)$. It is known that the algorithm advocated in the original CreditRisk + document in order to the probabilities, the Panjer recursion scheme, is numerically unstable. Giese(2003) has suggested the method to calculate the probabilities directly by applying standard algorithms for logarithm and exponential of power series. However, the numerical stability was analyzed by Haaf, Reiß and Schoenmakers(2004). An alternative algorithm using FFT(fast Fourier transform) is established by Rei $\beta(2004)$, which 
is described in terms of characteristic function instead of PGF. This algorithm is easy to implement and is numerically stable, so it may also be applied for large portfolios.

While the above researches have assumed independent risk factors, there are several researches considering the correlation between risk factors. Bürgisser, Kurth, Wagner and Wolf(1999) have proposed a model with a single factor approach to correlation, and Giese(2004) has discussed two multivariate factor distributions, multivariate gamma distribution and compound gamma distribution), which include risk factor correlations. However, using these methods for dealing with any correlations there is a limit.

\section{THE METHODOLOGY TO ESTIMATE THE INSURANCE LOSS DISTRIBUTION}

In this chapter we explain the algorithm to get the PGF for the loss distribution using CreditRisk + in each cases of one business line, independent business lines, and correlated business lines.

\subsection{A case of only one business line}

First, for a case of only one business line, the method for estimating the loss distribution of severity for given frequency are described. For this we have to calculate the PGF $F_{m}(z)$ of severity for $m$ events.

Suppose the severity distribution for $i$-th event of $m$ events is given as follows:

\begin{tabular}{|c|c|}
\hline Loss Level & Loss Probability \\
\hline$v_{1} L_{0}$ & $p_{1}^{i}$ \\
\hline$v_{2} L_{0}$ & $p_{2}^{i}$ \\
\hline$\vdots$ & $\vdots$ \\
\hline$v_{M} L_{0}$ & $p_{M}^{i}$ \\
\hline
\end{tabular}

Loss levels $v_{1}, v_{2}, \cdots, v_{M}$ are positive integers and $v_{1}<v_{2}<\cdots<v_{M}$. If each event is independent, the PGF of severity for $m$ events is

$$
F_{m}(z)=\prod_{i=1}^{m}\left\{1-\sum_{j=1}^{M} p_{j}^{i}+\sum_{j=1}^{M} p_{j}^{i} z^{v_{j}}\right\}=\prod_{i=1}^{m}\left\{1+\sum_{j=1}^{M} p_{j}^{i}\left(z^{v_{j}}-1\right)\right\}
$$

Also, if we assume $p_{j}^{1}=p_{j}^{2}=\cdots=p_{j}^{m}=p_{j}$ for $j=1,2, \cdots, M$, the PGF is represented by

$$
F_{m}(z)=\left\{1+\sum_{j=1}^{M} p_{j}\left(z^{v_{j}}-1\right)\right\}^{m} .
$$


Now, given frequency distribution, we have to estimate the loss distribution combined with severity distribution. Assume that frequency distribution is Poisson distribution with parameter $\lambda$. Then the PGF $G(z)$ of loss distribution is

$$
G(z)=\sum_{m=0}^{\infty} F_{m}(z) \frac{\lambda^{m}}{m !} e^{-\lambda}=\exp \left[\lambda\left\{\sum_{j=1}^{M} p_{j}\left(z^{v_{j}}-1\right)\right\}\right]
$$

\subsection{A case of independent business lines}

Assume that $N$ business lines are independent. Let $\lambda_{k}$ be the Poisson parameter of severity distribution for $k$-th business line and the severity distribution for $k$-th business line is given as follows:

\begin{tabular}{|c|c|}
\hline Loss Level & Loss Probability \\
\hline$v_{1}^{k} L_{0}$ & $p_{1}^{k}$ \\
\hline$v_{2}^{k} L_{0}$ & $p_{2}^{k}$ \\
\hline$\vdots$ & $\vdots$ \\
\hline$v_{M_{k}}^{k} L_{0}$ & $p_{M_{k}}^{k}$ \\
\hline
\end{tabular}

Loss levels $v_{1}^{k}, v_{2}^{k}, \cdots, v_{M_{k}}^{k}$ are positive integers and $v_{1}^{k}<v_{2}^{k}<\cdots<v_{M_{k}}^{k}$. Because the business lines are independent, using equation (1) the PGF $G(z)$ of $N$ business lines is represented by

$$
G(z)=\prod_{k=1}^{N} \exp \left[\lambda_{k}\left\{\sum_{j=1}^{M_{k}} p_{j}^{k}\left(z^{v_{j}^{k}}-1\right)\right\}\right]=\exp \left[\sum_{k=1}^{N} \lambda_{k}\left\{\sum_{j=1}^{M_{k}} p_{j}^{k}\left(z^{v_{j}^{k}}-1\right)\right\}\right] .
$$

We can assume that $M_{1}=M_{2}=\cdots=M_{N}=M$ without loss of generality, and if we assume that $v_{j}^{1}=v_{j}^{2}=\cdots=v_{j}^{N}=v_{j}$ for $j=1,2, \cdots, M$, then the PGF $G(z)$ of portfolio is briefly

$$
G(z)=\exp \left[\sum_{k=1}^{N} \lambda_{k}\left\{\sum_{j=1}^{M} p_{j}^{k}\left(z^{v_{j}}-1\right)\right\}\right] .
$$

3.3. A case of correlated business lines

In general, the events in each business line may correlate with each other. The correlation between business lines is explained by the correlation between the parameters $\lambda_{k}, k=1,2, \cdots, N$ of the frequency distribution: 


$$
C_{i j}=\operatorname{Corr}\left(\lambda_{i}, \lambda_{j}\right), i=1,2, \cdots, N, j=1,2, \cdots, N .
$$

We assume that this correlation matrix $C=\left(C_{i j}\right)$ is positive definite.

The correlation matrix $C=\left(C_{i j}\right)$ is decomposed into lower triangular matrix $L=\left(L_{i j}\right)$ and its transpose matrix by Cholesky decomposition $C=L L^{T}$, and there exist random variables $Y_{k}, k=1,2, \cdots, N$ independent of each other such that

$$
\left(\begin{array}{c}
\lambda_{1} \\
\lambda_{2} \\
\vdots \\
\lambda_{N}
\end{array}\right)=L\left(\begin{array}{c}
Y_{1} \\
Y_{2} \\
\vdots \\
Y_{N}
\end{array}\right) .
$$

Also, let $\mu_{k}=E\left(\lambda_{k}\right), \sigma_{k}=\sigma\left(\lambda_{k}\right)$ be the expectation and standard deviation of $\lambda_{k}$, and let $L^{-1}=\left(D_{i j}\right)$ be the inverse matrix of $L=\left(L_{i j}\right)$, then

$$
E\left(Y_{k}\right)=\sum_{i=1}^{N} D_{k i} \mu_{i}, \sigma^{2}\left(Y_{k}\right)=\sum_{i, j=1}^{N} D_{k i} D_{k j} \sigma_{i} \sigma_{j} C_{i j} .
$$

Let $f_{k}(z)=\sum_{j=1}^{M} p_{j}^{k}\left(z^{v_{j}}-1\right)$ and $\alpha_{k}=\frac{\left\{E\left(Y_{k}\right)\right\}^{2}}{\sigma^{2}\left(Y_{k}\right)}, \beta_{k}=\frac{\sigma^{2}\left(Y_{k}\right)}{E\left(Y_{k}\right)}$.

If the random variable $Y_{k}$ is gamma-distributed with parameters $\alpha_{k}, \beta_{k}$ and its probability density function is $g_{\alpha_{k}, \beta_{k}}$, then the PGF $G(z)$ of portfolio is calculated by

$$
\begin{aligned}
G(z) & =E\left[\exp \left\{\sum_{k=1}^{N} \lambda_{k} f_{k}(z)\right\}\right] \\
& =\int_{0}^{\infty} \cdots \int_{0}^{\infty} \exp \left[\sum_{k=1}^{N}\left\{\sum_{l=1}^{N} L_{k l} Y_{l}\right\} f_{k}(z)\right] g_{\alpha_{1}, \beta_{1}}\left(Y_{1}\right) \cdots g_{\alpha_{N}, \beta_{N}}\left(Y_{N}\right) d Y_{1} \cdots d Y_{N} \\
& =\prod_{l=1}^{N} \int_{0}^{\infty} \exp \left[Y_{l} \sum_{k=1}^{N} L_{k l} f_{k}(z)\right] g_{\alpha_{l}, \beta_{l}}\left(Y_{l}\right) d Y_{l} .
\end{aligned}
$$

The since $g_{\alpha, \beta}(y)=\frac{1}{\beta^{\alpha} \Gamma(\alpha)} e^{-\frac{y}{\beta}} y^{\alpha-1}$ and $\int_{0}^{\infty} e^{x y} \frac{1}{\beta^{\alpha} \Gamma(\alpha)} e^{-\frac{y}{\beta}} y^{\alpha-1} d y=(1-\beta x)^{-\alpha}$, then the PGF of portfolio is

$$
G(z)=\prod_{l=1}^{N}\left[1-\beta_{l} \sum_{k=1}^{N} L_{k l} f_{k}(z)\right]^{-\alpha_{l}}=\exp \left[-\sum_{l=1}^{N} \alpha_{l} \ln \left\{1-\beta_{l} \sum_{k=1}^{N} L_{k l} f_{k}(z)\right\}\right]
$$

\section{THE COMPUTING ALGORITHM OF LOSS DISTRIBUTION}


The methodology to compute the loss distribution from PGF functions has been well developed since CreditRisk + has been offered CSFP. The recursive algorithms by Panjer(1997) and Giese(2003), and the FFT method are mostly used and implemented by practitioners. The Panjer's method was firstly adopted and published in CreditRisk + by CSFP, but its instability is well known among credit risk managers so that we will explain Giese's algorithm(2003) and the FFT method briefly in this Chapter.

\subsection{Giese's Recursive Method}

Rewrite Equation (3) as follows.

$$
G(z)=\exp \left[-\sum_{l=1}^{N} \alpha_{l} \ln \left\{1+\beta_{l} \sum_{k=1}^{N} L_{k l} \sum_{i=1}^{M} p_{i}^{k}-\beta_{l} \sum_{k=1}^{N} L_{k l} \sum_{i=1}^{M} p_{i}^{k} z^{v_{i}}\right\}\right]
$$

Let $B$ be a pre-specified integer. Then, we can calculate the probability $p\left(\right.$ Loss $\left.\leq B L_{0}\right)$ that loss is equal or less than $B L_{0}$. The process consists of four steps as follows.

Step 1: Define $\quad l=1,2, \cdots, N$,

$$
a_{0}^{(l)}=1+\beta_{l} \sum_{k=1}^{N} L_{k l} \sum_{i=1}^{M} p_{i}^{k}, a_{j}^{(l)}=\beta_{l} \sum_{k=1}^{N} L_{k l} \sum_{i=1}^{M} p_{i}^{k} 1_{\left\{v_{i}=j\right\}}, j=1,2, \cdots, B,
$$

hence

$$
1+\beta_{l} \sum_{k=1}^{N} L_{k l} \sum_{i=1}^{M} p_{i}^{k}-\beta_{l} \sum_{k=1}^{N} L_{k l} \sum_{i=1}^{M} p_{i}^{k} z^{v_{i}}=a_{0}^{(l)}-\sum_{j=1}^{B} a_{j}^{(l)} z^{j}+O\left(z^{B+1}\right) .
$$

Thus

$$
G(z)=\exp \left[-\sum_{l=1}^{N} \alpha_{l} \ln \left\{a_{0}^{(l)}-\sum_{j=1}^{B} a_{j}^{(l)} z^{j}\right\}\right]+O\left(z^{B+1}\right),
$$

and the time required for this step is $O\left(N^{2} M B\right)$.

Step 2: Define $\quad l=1,2, \cdots, N$ ，

$$
b_{0}^{(l)}=-\ln a_{0}^{(l)}, b_{j}^{(l)}=\frac{1}{a_{0}^{(l)}}\left\{a_{j}^{(l)}+\frac{1}{j} \sum_{k=1}^{j-1} k b_{k}^{(l)} a_{j-k}^{(l)}\right\}, j=1,2, \cdots, B,
$$

hence

$$
-\ln \left\{a_{0}^{(l)}-\sum_{j=1}^{B} a_{j}^{(l)} z^{j}\right\}=\sum_{j=0}^{B} b_{j}^{(l)} z^{j}+O\left(z^{B+1}\right) .
$$

Thus

$$
G(z)=\exp \left[\sum_{l=1}^{N} \alpha_{l} \sum_{j=0}^{B} b_{j}^{(l)} z^{j}\right]+O\left(z^{B+1}\right)
$$


and the time required for this step is $O\left(N B^{2}\right)$.

Step 3: Define $\quad l=1,2, \cdots, N$,

hence

$$
c_{0}=\sum_{l=1}^{N} \alpha_{l} b_{0}^{(l)}, c_{j}=\sum_{l=1}^{N} \alpha_{l} b_{j}^{(l)}, j=1,2, \cdots, B,
$$

Thus

$$
\sum_{l=1}^{N} \alpha_{l} \sum_{j=0}^{B} b_{j}^{(l)} z^{j}=\sum_{j=0}^{B} c_{j} z^{j}+O\left(z^{B+1}\right) .
$$

$$
G(z)=\exp \left[\sum_{j=0}^{B} c_{j} z^{j}\right]+O\left(z^{B+1}\right)
$$

and the time required for this step is $O(N B)$.

Step 4: Define

$$
d_{j}=\exp \left(c_{0}\right), d_{j}=\sum_{i=1}^{j} \frac{i}{j} c_{i} d_{j-i}, j=1,2, \cdots, B
$$

Hence

$$
\exp \left[\sum_{j=0}^{B} c_{j} z^{j}\right]=\sum_{j=0}^{B} d_{j} z^{j}+O\left(z^{B+1}\right) .
$$

Thus

$$
G(z)=\sum_{j=0}^{B} d_{j} z^{j}+O\left(z^{B+1}\right)
$$

and the time required for this step is $O\left(B^{2}\right)$.

Therefore, for $j=0,1,2, \cdots, B$ the probability that loss equals $j L_{0}$ is $d_{j}$, i.e.

$$
p\left(\text { Loss }=j L_{0}\right)=d_{j} .
$$

\subsection{Fast Fourier Transform Method}

Using equation (3), the characteristic function of portfolio is

$$
\Phi(z)=G\left(e^{i z}\right)=\exp \left[-\sum_{l=1}^{N} \alpha_{l} \ln \left\{1-\beta_{l} \sum_{k=1}^{N} L_{k l} f_{k}\left(e^{i z}\right)\right\}\right] .
$$

Choose a number $B=2^{n}$ for some integer $n$. Let $\Lambda_{k}$ be the maximum loss of $k$-th business line and let

$$
z_{j}=(j-B / 2) \Delta z, x_{j}=j \Delta x, j=0,1,2, \cdots, B-1,
$$

where

$$
\Delta x=\sum_{k=1}^{N} \frac{\Lambda_{k}}{B-1}, \Delta z=\frac{2 \pi}{B \Delta x} .
$$

Then by Fourier inversion theorem the probability $p\left(\operatorname{loss}=x_{j}\right)$ is 


$$
p\left(\text { loss }=x_{j}\right)=\frac{1}{B} \sum_{k=0}^{B-1} e^{-2 \pi j k / B} \Phi\left(z_{k}\right) .
$$

The loss probability is obtained by the FFT algorithm and time required for this algorithm is $O\left(B \log _{2} B\right)$. For more information see Rei $\beta(2004)$. Compared with the recursive method, this algorithm is very simple and fast.

\section{DATA AND COMPUTATION RESULTS}

The sample data set is a causality insurance one provided by Korea Insurance Development Institute, which consists of monthly time series data for the time period between 2000, April and 2003, April collected according to insurance products. For fixed unit $L_{0} X 2048$ we rounded severities and frequencies data to display in Table 1 and 2 respectively.

In Table 2 we calculated the correlation matrix between each business line using monthly frequency time series data for the same time period. The "(Loss, Prob. $)=(14,0.00009)$ " in the first two columns of Table 1 means that the probability of loss event occurring by amount of $14 X L_{0} X 2048$ is 0.00009 . The second column in Table 2 means that the loss event of Fire business line occurs 3710 times in monthly average, and its standard deviation is 2338 , and that its correlations between each other business lines are $0.9212,0.9142$ and 0.8520 . Figure 1 represents the loss distribution estimated from the data in Table 1 and 2 using equation (3).

Table 1. Severity Data

\begin{tabular}{|c|c|c|c|c|c|c|c|}
\hline \multicolumn{2}{|c|}{ Fire } & \multicolumn{2}{c|}{ Marine } & \multicolumn{2}{c|}{ Liability } & \multicolumn{2}{c|}{ Comprehensive } \\
\hline Loss & Prob. & Loss & Prob. & Loss & Prob. & Loss & Prob. \\
\hline 14 & 0.00009 & 8 & 0.00013 & 2 & 0.00055 & 2 & 0.00216 \\
\hline 17 & 0.00013 & 9 & 0.00016 & 3 & 0.00401 & 3 & 0.00323 \\
\hline 23 & 0.00117 & 10 & 0.00041 & 4 & 0.07564 & 4 & 0.01866 \\
\hline 24 & 0.00198 & 11 & 0.00005 & 5 & 0.02706 & 5 & 0.02570 \\
\hline 25 & 0.00072 & 12 & 0.00069 & & & 6 & 0.07943 \\
\hline 26 & 0.00198 & 13 & 0.00058 & & & 7 & 0.06626 \\
\hline 27 & 0.00189 & 14 & 0.00067 & & & 8 & 0.02789 \\
\hline 28 & 0.00117 & 15 & 0.00119 & & & 9 & 0.00401 \\
\hline 29 & 0.00072 & 16 & 0.00192 & & & 11 & 0.00265 \\
\hline 30 & 0.00037 & 17 & 0.00028 & & & & \\
\hline 31 & 0.00118 & 23 & 0.00003 & & & & \\
\hline 32 & 0.00004 & & & & & & \\
\hline
\end{tabular}


Table 2. Frequency Data

\begin{tabular}{|c|c|r|r|r|r|}
\hline & & \multicolumn{1}{c|}{ Fire } & \multicolumn{1}{c|}{ Marine } & \multicolumn{1}{c|}{ Liability } & \multicolumn{1}{c|}{ Comp. } \\
\hline \multicolumn{2}{|c|}{ Average } & 3710 & 6226 & 13890 & 19283 \\
\hline \multirow{4}{*}{ Corr. } & St. Dev. & 2338 & 3654 & 8230 & 11837 \\
\cline { 2 - 6 } & Fire & 1.0000 & 0.9212 & 0.9142 & 0.8520 \\
\cline { 2 - 6 } & Marine & 0.9212 & 1.0000 & 0.9775 & 0.9795 \\
\cline { 2 - 6 } & Liability & 0.9142 & 0.9775 & 1.0000 & 0.9774 \\
\cline { 2 - 6 } & Comp. & 0.8520 & 0.9795 & 0.9774 & 1.0000 \\
\hline
\end{tabular}

Figure 1. Loss Distribution

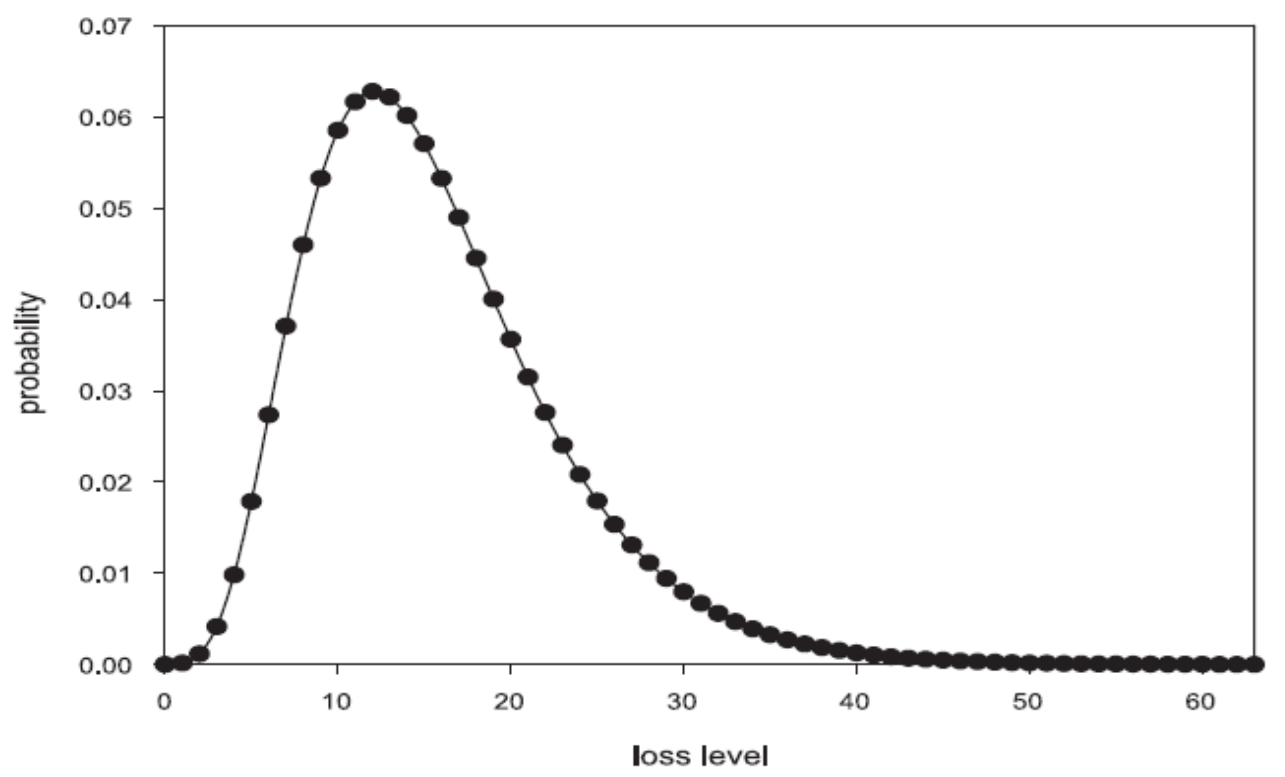

It takes much time to calculate the loss distribution by the recursive method of Giese (2003) because we should do it by $B=2^{17}$. On the contrary, the FFT method demands $O\left(B \log _{2} B\right)$ in computation time so we adopt the FFT method. We also used the recursive method of Panjer (1997, CSFP) to estimate the loss distribution but had the very unstable results. The loss level $v_{i}$ in Figure 1 is an approximation to the actual exposed loss $\varepsilon_{i}$, i.e.,

$$
\varepsilon_{i} \approx 2048 \times v_{i} L_{0} \text {. }
$$

In other words it is different from the loss level $v_{i}$ defined in Chapter 3 by 2048.

And we see that 


$$
\operatorname{VaR}_{q}=37.22 \text { when } \mathrm{q}=99 \% \text {, }
$$

and

$$
\operatorname{VaR}_{q}=48.38 \text { when } \mathrm{q}=99.9 \% \text {. }
$$

We note that the loss distribution has shape similar to a typical credit loss distribution with long-tailed implying the loss event being rare one.

\section{CONCLUSION}

In this paper we offered an efficient algorithm for causality insurance company to measure an insurance risk using CreditRisk+ methodology. In particular we proposed a mathematical method to get the loss distribution in case of correlated business lines, explained an algorithm to implement it effectively, and estimated the loss distribution and the VaR with real data.

We could compute the CreditVaR to get speedy and robust results. It is because we adopted an analytic approximation to generate the probability generating function. Even though it is a nonparametric method its input data type is so simple. For example, we estimated correlations among severities of business lines with serial data and put them in the model directly. In other words, the model allows the simplest manner of estimating correlations. The CreditRisk+ assumes independent sectors which is too strong for practitioners to accommodate for practical applications such as capital allocation and RAPM. Our model avoids the assumptions of independence among business lines. As we see in Figure 1 we know that the aggregated loss distribution estimated with our algorithm displays a typical shape of a loss distribution.

The insurance company can get the aggregated VaR in terms of credit risks and insurance risks with our method, which enables the risk manager to use the VaR as the firm-wide total risk.

Our future research direction will be to find a way to apply the estimated loss distribution to DFA, and to get the actual aggregated loss distribution of the portfolio containing loans and bonds with credit risks and insurance products with insurance risks.

\section{REFERENCES}

[1] Akkaya, N., A.Kurth and A.Wagner, "Incorporating Default Correaltions and Severity Variations" CreditRisk+ in the Banking Industry, Springer-Verlag, 2004,129-152

[2] Bürgisser, P., A. Kurth, A.Wagner and M.Wolf, “Integrating Correlations”, Risk, 12,1999, 57-60

[3] Credit Suisse Financial Products, "CreditRisk+: A Credit Risk Management Framework", London, 1997

[4] Giese, G., "Dependent Risk Factors", CreditRisk+ in the Banking Industry, Springer-Verlag, 2004, 153-165

[5] Giese, G., "Enhancing CreditRisk+", Risk, 16, 2003, 73-77

[6 ] Haaf, H., O. Rei $\beta$ and J. Schoenmakers, "Numerically Stable Computation of CreditRisk+" CreditRisk+ in the Banking Industry, Springer-Verlag, 2004, 69-77

[7] Heckman, P. E. and G.G. Meyers, "The Calculation of Aggregate Loss Distribution from Claim Severity and Claim Count Distributions", PCSA LXX, 1983, 22-61

[8] Rei $\beta$, O., "Fourier Inversion Techniques for CreditRisk+", CreditRisk+ in the Banking Industry, SpringerVerlag, 2004, 111-128 
[9] Rovertson, J., "The Computation of Aggregate Loss Distribution", PCSA LXXIX, 1992, 57-133

[10] Wang, S. S., "Aggregation of Correlated Risk Portfolios: Models and Algorithms", The proceeding of Casuality Actuarial Society LXXXV, 1998, 781-805 